\title{
Global Asymptotic Stability of Traveling Waves in Delayed Reaction-Diffusion Equations
}

\author{
April 20, 1999 \\ Hal L. Smith ${ }^{1}$ and Xiao-Qiang Zhao \\ Department of Mathematics \\ Arizona State University \\ Tempe, AZ 85287-1804, USA
}

Key words. Delayed reaction-diffusion equations, comparison principle, super- and sub-solutions, traveling waves.

AMS(MOS) Subject Classifications: 35R10, 35B40, 34K30, 58D25.

\begin{abstract}
The existence and comparison theorem of solutions is first established for the quasimonotone delayed reaction-diffusion equations on $R$ by appealing to the theory of abstract functional differential equations. The global asymptotic stability, Liapunov stability and uniqueness of traveling wave solutions are then proved by the elementary super- and sub-solution comparison and squeezing methods.
\end{abstract}

\footnotetext{
${ }^{1}$ Supported by NSF Grant DMS 9700910.
} 


\section{Introduction}

Traveling wave solutions have been widely studied for nonlinear reactiondiffusion equations modeling a variety of physical and biological phenomena(see, e.g., [3], [11], monograph [16] and references therein). More recently, Chen [1] studied the existence, uniqueness and global asymptotic stability of traveling wave solutions in nonlocal evolution equations with bistable nonlinearities. A basic assumption in [1] is the comparison principle. Shen [12] investigated these problems for traveling wave solutions in temporally almost periodic reaction-diffusion equations with bistable nonlinearities. In [9], Ogiwara and Matano discussed the monotonicity and stability of pseudotraveling wave solutions in temporally or spatially periodic media, as an application of their general theory on stable equilibria in order-preserving systems in the presence of symmetry.

Recently, great attention has also been paid to reaction-diffusion equations with time delays(see, e.g., [15], [7], [8], [4], monograph [17] and references therein). Most of the known results in this direction are about the existence, comparison, monotonicity, bifurcations and asymptotic behavior of solutions to delayed reaction-diffusion equations on a bounded spatial domain. Schaaf [13] first studied traveling wave solutions for some delayed reaction-diffusion equations and, in particular, proved the existence of monotone traveling wave solutions and uniqueness of wave speeds for the delayed reaction-diffusion equations with quasimonotone and bistable nonlinearities by a phase plane analysis method. In [18], Zou and Wu obtained the existence of traveling waves in some delayed reaction-diffusion systems via the monotone iteration method. As a consequence of the delayed reaction term, the study of uniqueness and global asymptotic stability of traveling wave solutions becomes relatively more difficult. This paper is devoted to the study of global asymptotic stability with phase shift, Liapunov stability and uniqueness up to translation of traveling wave solutions in delayed reactiondiffusion equations with quasimonotone and bistable nonlinearities. The first key point is to establish a refined comparison principle for this class of delayed reaction-diffusion equations defined on the whole real line $R$. We do this by appealing to the theory of abstract functional differential equations developed in [7] and properties of the analytic semigroup generated by the one-dimensional Laplacian operator on the Banach space of all bounded and

uniformly continuous functions on $R$. In order to prove global asymptotic stability of monotone traveling wave solutions, we have borrowed a "squeez- 
ing" technique introduced in [1], which is similar in spirit to a "contracting rectangles" approach developed in [8] for quasimonotone delayed reactiondiffusion systems on a bounded spatial domain. The Liapunov stability of monotone traveling waves and the uniqueness of traveling waves are then proved by using an elementary super- and sub-solution comparison method and the established global asymptotic stability of monotone traveling waves.

We note that the recent publication [9] contains results related to ours on traveling wave solutions for delayed reaction-diffusion equations. These authors show that monotone traveling waves are locally asymptotically stable with phase shift. By contrast, our results allow replacing 'locally' with globally and exponentially in their result and are based on more elementary methods.

The organization of this paper is as follows. In section 2, we establish an existence and comparison theorem for quasimonotone delayed reactiondiffusion equations on $R$ (Theorem 2.1). For the use in next section, we also prove three technical lemmas about the construction of super- and sub-solutions and the derivative of profiles of monotone traveling wave solutions(Lemmas 2.1, 2.2 and 2.3). In section 3, we first prove two lemmas about the iteration and ultimate estimation of solutions(Lemmas 3.1 and 3.2), then we establish the global exponential stability with phase shift of monotone traveling wave solutions(Theorem 3.1), Liapunov stability and uniqueness up to translation of traveling wave solutions (Theorem 3.2).

Acknowledgement: The authors would like to thank Professor H. Matano for valuable discussions during his visit to the Arizona State University.

\section{Existence and comparison of solutions}

Consider delayed reaction-diffusion equations

$$
\begin{cases}\frac{\partial u}{\partial t} & =d \Delta u+f(u(x, t), u(x, t-\tau)), \quad x \in R, t>0 \\ u(x, s) & =\varphi(x, s), \quad x \in R, s \in[-\tau, 0],\end{cases}
$$

where $d>0, \tau \geq 0$, and $\Delta$ is the Laplacian operator on $R$. We will impose the following conditions on $f(\cdot, \cdot)$. 
(H1) $f \in C^{1}\left(I^{2}, R\right)$ for some open interval $I \subset R$ with $[0,1] \subset I ; \partial_{2} f(u, v) \geq$ 0 , for $(u, v) \in I^{2}$.

(H2) $f(0,0)=f(1,1)=0, \partial_{1} f(0,0)+\partial_{2} f(0,0)<0$, and $\partial_{1} f(1,1)+\partial_{2} f(1,1)<$ 0 .

Let $X=B U C(R, R)$ be the Banach space of all bounded and uniformly continuous functions from $R$ into $R$ with the usual supremum norm. Let $X^{+}=\{\varphi \in X ; \varphi(x) \geq 0, x \in R\}$. It is easy to see that $X^{+}$is a closed cone of $X$ and its induced partial ordering makes $X$ into a Banach lattice. By [2, Theorem 1.5], it then follows that the $X$-realization $d \Delta_{X}$ of $d \Delta$ generates a strongly continuous analytic semigroup $T(t)$ on $X$ and $T(t) X^{+} \subset X^{+}, t \geq 0$. Moreover, by the explicit expression of solutions of the heat equation

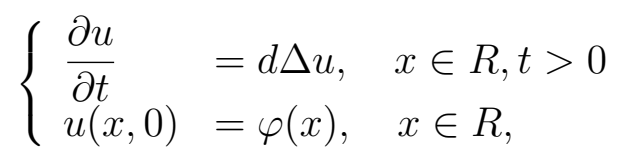

we have

$$
\begin{gathered}
T(t) \varphi(x)=\frac{1}{\sqrt{4 \pi d t}} \int_{-\infty}^{\infty} \exp \left(-\frac{(x-y)^{2}}{4 d t}\right) \varphi(y) d y, \\
x \in R, \quad t>0, \quad \varphi(\cdot) \in X .
\end{gathered}
$$

Let $f_{0}(\cdot): I \rightarrow R$ be defined by $f_{0}(u)=f(u, u), u \in I$. By the continuity of $f_{0}$ and condition (H2), it then easily follows that there exist $\delta_{0}, a^{-}, a^{+} \in$ $(0,1)$ with $\left[-\delta_{0}, 1+\delta_{0}\right] \subset I$ and $a^{-} \leq a^{+}$such that $f_{0}(\cdot):\left[-\delta_{0}, 1+\delta_{0}\right] \rightarrow R$ satisfies

$$
\begin{aligned}
& f_{0}(0)=f_{0}\left(a^{-}\right)=f_{0}\left(a^{+}\right)=f_{0}(1)=0, \\
& f_{0}(u)>0 \text { for } u \in\left[-\delta_{0}, 0\right) \cup\left(a^{+}, 1\right), \\
& \text { and } f_{0}(u)<0 \text { for } u \in\left(0, a^{-}\right) \cup\left(1,1+\delta_{0}\right] .
\end{aligned}
$$

Let $L_{i}=\max \left\{\left|\partial_{i} f(u, v)\right| ;-\delta_{0} \leq u, v \leq 1+\delta_{0}\right\}, i=1,2$, and define

$$
\theta(J, t)=\frac{1}{\sqrt{4 \pi d t}} \exp \left(-L_{1} t-\frac{(J+1)^{2}}{4 d t}\right), \quad J \geq 0, t>0 .
$$

Clearly, $\theta \in C([0, \infty) \times(0, \infty), R)$.

Let $C=C([-\tau, 0], X)$ be the Banach space of continuous functions from $[-\tau, 0]$ into $X$ with the supremum norm, and let $C^{+}=\{\varphi \in C ; \varphi(s) \in$ 
$\left.X^{+}, s \in[-\tau, 0]\right\}$. Then $C^{+}$is a positive cone of $C$. For convenience, we identify an element $\varphi \in C$ as a function from $R \times[-\tau, 0]$ into $R$ defined by $\varphi(x, s)=(\varphi(s))(x)$. For any continuous function $w(\cdot):[-\tau, b) \rightarrow X, b>0$, we define $w_{t} \in C, t \in[0, b)$, by $w_{t}(s)=w(t+s), s \in[-\tau, 0]$. It is then easy to see that $t \mapsto w_{t}$ is a continuous function from $[0, b)$ to $C$. For any $\varphi \in\left[-\delta_{0}, 1+\delta_{0}\right]_{C}=\left\{\varphi \in C ; \varphi(x, s) \in\left[-\delta_{0}, 1+\delta_{0}\right], x \in R, s \in[-\tau, 0]\right\}$, define $F(\varphi)(x)=f(\varphi(x, 0), \varphi(x,-\tau)), x \in R$. By the global Lipschitz continuity of $f(\cdot, \cdot)$ on $\left[-\delta_{0}, 1+\delta_{0}\right]^{2}$, we can verify that $F(\varphi) \in X$ and $F:\left[-\delta_{0}, 1+\delta_{0}\right]_{C} \rightarrow$ $X$ is globally Lipschitz continuous.

Definition 2.1 A continuous function $v:[-\tau, b) \rightarrow X, b>0$, is called $a$ super-solution (sub-solution) of (2.1) on $[0, b)$ if

$$
v(t) \geq(\leq) T(t-s) v(s)+\int_{s}^{t} T(t-r) F\left(v_{r}\right) d r
$$

for all $0 \leq s<t<b$. If $v$ is both a super-solution and a sub-solution on $[0, b)$, then we call it a mild solution of (2.1).

Remark 2.1 Assume that there is a $v \in B U C(R \times[-\tau, b], R), b>0$, such that $v$ is $C^{2}$ in $x \in R, C^{1}$ in $t \in(0, b)$ and

$$
\frac{\partial v}{\partial t} \geq(\leq) d \Delta v+f(v(x, t), v(x, t-\tau)), \quad x \in R, t \in(0, b) .
$$

Then, by the positivity of the linear semigroup $T(t): X \rightarrow X$, it easily follows that (2.5) holds. Hence $v$ is a super-solution (sub-solution) of (2.1) on $[0, b)$.

Now we are in a position to establish the following existence and comparison theorem for (2.1).

Theorem 2.1 Assume that (H1) and (H2) hold. Then for any $\varphi \in\left[-\delta_{0}, 1+\right.$ $\left.\delta_{0}\right]_{C}$, equation (2.1) has a unique mild solution $u(x, t, \varphi)$ on $[0, \infty)$ and $u(x, t, \varphi)$ is a classical solution to (2.1) for $(x, t) \in R \times(\tau, \infty)$. Furthermore, for any pair of super-solution $u(x, t)$ and sub-solution $w(x, t)$ of (2.1) on $[0, \infty)$ with $-\delta_{0} \leq u(x, t), w(x, t) \leq 1+\delta_{0}, x \in R, t \in[-\tau, \infty)$, and $u(x, s) \geq w(x, s)$, $x \in R, s \in[-\tau, 0]$, there holds $u(x, t) \geq w(x, t)$ for $x \in R, t \geq 0$, and

$$
u(x, t)-w(x, t) \geq \theta\left(J, t-t_{0}\right) \int_{z}^{z+1}\left(u\left(y, t_{0}\right)-w\left(y, t_{0}\right)\right) d y
$$

for any $J \geq 0, x$ and $z \in R$ with $|x-z| \leq J$, and $t>t_{0} \geq 0$. 
Proof. Under an abstract setting in [7], a mild solution of (2.1) is a solution to its associated integral equation

$$
\left\{\begin{array}{l}
u(t)=T(t) \varphi(0)+\int_{0}^{t} T(t-r) F\left(u_{r}\right) d r, \quad t>0 \\
u_{0}=\varphi \in\left[-\delta_{0}, 1+\delta_{0}\right]_{C} .
\end{array}\right.
$$

By the choice of $\delta_{0}$ in (2.4), we have $f\left(1+\delta_{0}, 1+\delta_{0}\right)<0$ and $f\left(-\delta_{0},-\delta_{0}\right)>0$. Clearly, $v^{+}=1+\delta_{0}$ and $v^{-}=-\delta_{0}$ are an ordered pair of super- and subsolutions of $(2.1)$ on $[0, \infty)$. As aforementioned, $F:\left[-\delta_{0}, 1+\delta_{0}\right]_{C} \rightarrow X$ is globally Lipschitz continuous. We further claim that $F$ is quasi-monotone on $\left[-\delta_{0}, 1+\delta_{0}\right]_{C}$ in the sense that

$$
\begin{gathered}
\lim _{h \rightarrow 0^{+}} \frac{1}{h} \operatorname{dist}\left(\psi(0)-\varphi(0)+h[F(\psi)-F(\varphi)] ; X^{+}\right)=0 \\
\quad \text { for all } \psi, \varphi \in\left[-\delta_{0}, 1+\delta_{0}\right]_{C} \text { with } \psi \geq \varphi
\end{gathered}
$$

Indeed it follows from condition (H1) that

$$
\begin{aligned}
F(\psi)-F(\varphi) & =f(\psi(\cdot, 0), \psi(\cdot,-\tau))-f(\varphi(\cdot, 0), \varphi(\cdot,-\tau)) \\
& \geq f(\psi(\cdot, 0), \varphi(\cdot,-\tau))-f(\varphi(\cdot, 0), \varphi(\cdot,-\tau)) \\
& \geq-L_{1}(\psi(0)-\varphi(0)) \quad \text { in } X
\end{aligned}
$$

and hence, for any $h>0$ satisfying $h L_{1}<1$,

$$
\psi(0)-\varphi(0)+h[F(\psi)-F(\varphi)] \geq\left(1-L_{1} h\right)(\psi(0)-\varphi(0)) \geq 0 \quad \text { in } X .
$$

Then the existence and uniqueness of $u(x, t, \varphi)$ follows from [7, Corollary 5] with $S(t, s)=T(t, s)=T(t-s), t \geq s \geq 0$, and $B(t, \varphi) \equiv F(\varphi)$. Moreover, by a semigroup theory argument given in the proof of [7, Theorem 1], it follows that $u(x, t, \varphi)$ is a classical solution for $t>\tau$.

For simplicity, let $\psi(x, s)=u(x, s), \varphi(x, s)=w(x, s), x \in R, s \in[-\tau, 0]$. Then $\psi, \varphi \in\left[-\delta_{0}, 1+\delta_{0}\right]_{C}$ with $\psi \geq \varphi$ in $C$. Again by [7, Corollary 5], we have

$$
1+\delta_{0} \geq u(x, t, \psi) \geq u(x, t, \varphi) \geq-\delta_{0}, \quad x \in R, t \geq 0 .
$$

By applying [7, Corollary 5] with $v^{+}(x, t)=1+\delta_{0}$ and $v^{-}(x, t)=w(x, t)$, $v^{+}(x, t)=u(x, t)$ and $v^{-}(x, t)=-\delta_{0}$, respectively, we get

$$
w(x, t) \leq u(x, t, \varphi) \leq 1+\delta_{0}, \quad x \in R, t \geq 0,
$$


and

$$
-\delta_{0} \leq u(x, t, \psi) \leq u(x, t), \quad x \in R, t \geq 0 .
$$

Combining (2.10)-(2.12), we have $u(x, t) \geq w(x, t)$ for all $x \in R$ and $t \geq 0$.

It remains to prove the last inequality in the theorem. Let $v(x, t)=$ $u(x, t)-w(x, t), x \in R, t \in[-\tau, \infty)$. Then $v(x, t) \geq 0, x \in R, t \in[-\tau, \infty)$. Clearly, $u_{t}, w_{t} \in\left[-\delta_{0}, 1+\delta_{0}\right]_{C}$ with $u_{t} \geq w_{t}$ in $C$ for all $t \geq 0$. For any given $t_{0} \geq 0$, by Definition 2.1 and (2.9), it then follows that, for all $t \geq t_{0}$,

$$
\begin{aligned}
v(t) & \geq T\left(t-t_{0}\right) v\left(t_{0}\right)+\int_{t_{0}}^{t} T(t-r)\left(F\left(u_{r}\right)-F\left(w_{r}\right)\right) d r \\
& \geq T\left(t-t_{0}\right) v\left(t_{0}\right)-L_{1} \int_{t_{0}}^{t} T(t-r) v(r) d r
\end{aligned}
$$

Let

$$
z(t)=e^{-L_{1}\left(t-t_{0}\right)} T\left(t-t_{0}\right) v\left(t_{0}\right), \quad t \geq t_{0} .
$$

Then $z(t)$ satisfies

$$
z(t)=T\left(t-t_{0}\right) z\left(t_{0}\right)-L_{1} \int_{t_{0}}^{t} T(t-r) z(r) d r, \quad t \geq t_{0} .
$$

By [7, Proposition 3] with $v^{-} \equiv z(t), v^{+}=+\infty, S(t, s)=S^{-}(t, s)=$ $T(t, s)=T(t-s), t \geq s \geq 0$, and $B(t, \varphi) \equiv B^{-}(t, \varphi) \equiv-L_{1} \varphi(0)$, we have $v(t) \geq z(t)$ for all $t \geq t_{0}$. Thus it follows that

$$
u(t)-w(t) \geq e^{-L_{1}\left(t-t_{0}\right)} T\left(t-t_{0}\right)\left(u\left(t_{0}\right)-w\left(t_{0}\right)\right), \quad t \geq t_{0} .
$$

Combining $(2.3),(2.15)$ and the definition of $\theta \in C([0, \infty) \times(0, \infty), R)$, we then have

$$
u(x, t)-w(x, t) \geq \theta\left(J, t-t_{0}\right) \int_{z}^{z+1}\left(u\left(y, t_{0}\right)-w\left(y, t_{0}\right)\right) d y
$$

for all $x \in R$ with $|x-z| \leq J$ and $t>t_{0} \geq 0$.

This completes the proof.

For delayed reaction-diffusion equation (2.1), we are interested in its traveling wave solutions which connect two equilibria 0 and 1 . Without loss of generality, throughout this paper, by a traveling wave we refer to a solution of the form of $u(x, t)=U(x-c t), x \in R, t \in R$, with the property that

$$
\lim _{\xi \rightarrow \infty} U(\xi)=1, \quad \lim _{\xi \rightarrow-\infty} U(\xi)=0,
$$


where $U(\xi)$ is a function on $R$ and $c$ is a constant real number. As usual, $|c|$ is called the wave speed and $U$ the profile of the wave front. Moreover, we say a traveling wave $U(x-c t)$ is a monotone if $U(\cdot): R \rightarrow R$ is a strictly increasing function.

Lemma 2.1 Assume that (H1) and (H2) hold and let $U(x-c t)$ be a monotone traveling wave solution. Then there exist three positive numbers $\beta_{0}$ (which is independent of $U$ ), $\sigma_{0}$ and $\bar{\delta}$ such that for any $\delta \in(0, \bar{\delta}]$ and every $\xi_{0} \in R$, the functions $w^{+}$and $w^{-}$defined by

$$
w^{ \pm}(x, t):=U\left(x-c t+\xi_{0} \pm \sigma_{0} \delta\left[1-e^{-\beta_{0} t}\right]\right) \pm \delta e^{-\beta_{0} t}
$$

are a super-solution and a sub-solution of (2.1) on $[0, \infty)$, respectively.

Proof. Clearly, $0<U(\xi)<1$ and hence $0<U(x-c t)<1, x \in R, t \in R$. By Theorem 2.1 and monotonicity of $U(\cdot)$, it follows that $U(\cdot) \in C^{1}(R)$ and $U^{\prime}(\xi)>0, \xi \in R$. Since

$$
\lim _{(u, v, \beta) \rightarrow(0,0,0)}\left(\partial_{1} f(u, v)+e^{\beta \tau} \partial_{2} f(u, v)+\beta\right)=\partial_{1} f(0,0)+\partial_{2} f(0,0)<0
$$

and

$$
\lim _{(u, v, \beta) \rightarrow(1,1,0)}\left(\partial_{1} f(u, v)+e^{\beta \tau} \partial_{2} f(u, v)+\beta\right)=\partial_{1} f(1,1)+\partial_{2} f(1,1)<0,
$$

we can fix $\beta_{0}>0$ and $\delta^{*} \in\left(0, \delta_{0}\right)$ such that

$\partial_{1} f(u, v)+e^{\beta_{0} \tau} \partial_{2} f(u, v)<-\beta_{0}, \quad$ for all $(u, v) \in\left[-\delta^{*}, \delta^{*}\right]^{2} \cup\left[1-\delta^{*}, 1+\delta^{*}\right]^{2}$.

Let $c_{0}=\tau|c|+\left(e^{\beta_{0} \tau}-1\right)$. By $(2.17)$, there exists $M_{0}=M_{0}\left(U, \beta_{0}, \delta^{*}\right)>0$ such that

$$
\begin{aligned}
& U(\xi) \geq 1-\frac{\delta^{*}}{2}, \quad \text { for all } \xi \geq M_{0}-c_{0}, \\
& U(\xi) \leq \frac{\delta^{*}}{2}, \quad \text { for all } \xi \leq-M_{0}+c_{0} .
\end{aligned}
$$

Take

$$
\begin{aligned}
c_{1}= & c_{1}\left(\beta_{0}, \delta^{*}\right)=\max \left\{\left|\partial_{1} f(u, v)\right| ;(u, v) \in\left[-\delta^{*}, 1+\delta^{*}\right]^{2}\right\} \\
& +e^{\beta_{0} \tau} \max \left\{\left|\partial_{2} f(u, v)\right| ;(u, v) \in\left[-\delta^{*}, 1+\delta^{*}\right]^{2}\right\},
\end{aligned}
$$


$m_{0}=m_{0}\left(U, \beta_{0}, \delta^{*}\right)=\min \left\{U^{\prime}(\xi) ;|\xi| \leq M_{0}\right\}>0$, and define

$\sigma_{0}=\sigma_{0}\left(U, \beta_{0}, \delta^{*}\right)=\frac{\beta_{0}+c_{1}}{m_{0} \beta_{0}}>0, \bar{\delta}=\bar{\delta}\left(U, \beta_{0}, \delta^{*}\right)=\min \left\{\frac{1}{\sigma_{0}}, \frac{\delta^{*}}{2} \cdot e^{-\beta_{0} \tau}\right\}>0$.

We only prove $w^{+}(x, t)$ is a super-solution of (2.1) since the proof for $w^{-}(x, t)$ is analogous. By a translation, we can assume that $\xi_{0}=0$. For any given $\delta \in(0, \bar{\delta}]$, let $\xi(t)=x-c t+\sigma_{0} \delta\left[1-e^{-\beta_{0} t}\right]$. It then follows that $\xi(t)+c \tau \geq$ $\xi(t-\tau)$ and $|\xi(t-\tau)-\xi(t)|=\left|c \tau+\sigma_{0} \delta e^{-\beta_{0} t}\left[1-e^{\beta_{0} \tau}\right]\right| \leq c_{0}$ for all $t \geq 0$. Since $U(x-c t)$ is a solution of $(2.1)$, we have

$$
d U^{\prime \prime}(\xi)+c U^{\prime}(\xi)+f(U(\xi), U(\xi+c \tau))=0, \xi \in R
$$

Notice that $U^{\prime}(\xi)>0$ and $\partial_{2} f(\cdot, \cdot) \geq 0$. It then follows that, for any $t \geq 0$,

$$
\begin{aligned}
& \frac{\partial w^{+}(x, t)}{\partial t}-d \Delta w^{+}(x, t)-f\left(w^{+}(x, t), w^{+}(x, t-\tau)\right) \\
&=U^{\prime}(\xi(t))\left(-c+\sigma_{0} \delta \beta_{0} e^{-\beta_{0} t}\right)-\beta_{0} \delta e^{-\beta_{0} t} \\
& \quad-d U^{\prime \prime}(\xi(t))-f\left(U(\xi(t))+\delta e^{-\beta_{0} t}, U(\xi(t-\tau))+\delta e^{-\beta_{0}(t-\tau)}\right) \\
&=\left(U^{\prime}(\xi(t)) \sigma_{0} \beta_{0}-\beta_{0}\right) \delta e^{-\beta_{0} t}+f(U(\xi(t)), U(\xi(t)+(c \tau))) \\
& \quad-f\left(U(\xi(t))+\delta e^{-\beta_{0} t}, U\left(\xi(t-\tau)+\delta e^{-\beta_{0}(t-\tau)}\right)\right. \\
& \geq\left(U^{\prime}(\xi(t)) \sigma_{0} \beta_{0}-\beta_{0}\right) \delta e^{-\beta_{0} t}+f(U(\xi(t)), U(\xi(t-\tau))) \\
&-f\left(U(\xi(t))+\delta e^{-\beta_{0} t}, U(\xi(t-\tau))+\delta e^{-\beta_{0}(t-\tau)}\right) \\
&= \delta e^{-\beta_{0} t}\left[U^{\prime}(\xi(t)) \sigma_{0} \beta_{0}-\beta_{0}-\int_{0}^{1}\left(\partial _ { 1 } f \left(U(\xi(t))+s \delta e^{-\beta_{0} t}, U(\xi(t-\tau))\right.\right.\right. \\
&\left.\left.\left.+s \delta e^{-\beta_{0}(t-\tau)}\right)+e^{\beta_{0} \tau} \partial_{2} f\left(U(\xi(t))+s \delta e^{-\beta_{0} t}, U(\xi(t-\tau))+s \delta e^{-\beta_{0}(t-\tau)}\right)\right) d s\right] .
\end{aligned}
$$

We distinguish among three cases:

Case (i) $|\xi(t)| \leq M_{0}$ : By the choice of $M_{0}$ and $c_{1}$, the absolute value of the integral in (2.21) is less than or equal to $c_{1}$. Then, by the choice of $\sigma_{0}$, we have

$$
\begin{aligned}
& \frac{\partial w^{+}(x, t)}{\partial t}-d \Delta w^{+}(x, t)-f\left(w^{+}(x, t), w^{+}(x, t-\tau)\right) \\
& \geq\left[m_{0} \sigma_{0} \beta_{0}-\beta_{0}-c_{1}\right] \cdot \delta e^{-\beta_{0} t}=0 .
\end{aligned}
$$

Case (ii) $\xi(t) \geq M_{0}$ : Clearly, $\xi(t-\tau)=\xi(t)+\xi(t-\tau)-\xi(t) \geq M_{0}-c_{0}$. Then, by $(2.19), 1-\frac{\delta^{*}}{2} \leq U(\xi(t)), U(\xi(t-\tau)) \leq 1$. Thus we have

$$
1-\frac{\delta^{*}}{2} \leq U(\xi(t))+\delta e^{-\beta_{0} t} \leq 1+\frac{\delta^{*}}{2}
$$


and

$$
1-\frac{\delta^{*}}{2} \leq U(\xi(t-\tau))+\delta e^{-\beta_{0}(t-\tau)} \leq 1+\delta e^{\beta_{0} \tau} \cdot e^{-\beta_{0} t} \leq 1+\frac{\delta^{*}}{2} .
$$

Therefore, by (2.21) and (2.18), it follows that

$$
\begin{gathered}
\frac{\partial w^{+}(x, t)}{\partial t}-d \Delta w^{+}(x, t)-f\left(w^{+}(x, t), w^{+}(x, t-\tau)\right) \\
\geq\left[U^{\prime}(\xi(t)) \sigma_{0} \beta_{0}-\beta_{0}-\left(-\beta_{0}\right)\right] \delta e^{-\beta_{0} t} \geq 0 .
\end{gathered}
$$

Case (iii) $\xi(t) \leq-M_{0}$ : Clearly, $\xi(t-\tau) \leq \xi(t)+\xi(t-\tau)-\xi(t) \leq-M_{0}+$ $c_{0}$. Then, again by $(2.19), 0 \leq U(\xi(t)), U(\xi(t-\tau)) \leq \frac{\delta^{*}}{2}$, and hence

$$
0 \leq U(\xi(t))+\delta e^{-\beta_{0} t} \leq \frac{\delta^{*}}{2}+\delta e^{-\beta_{0} t} \leq \delta^{*}
$$

and

$$
0 \leq U(\xi(t-\tau))+\delta e^{-\beta_{0}(t-\tau)} \leq \frac{\delta^{*}}{2}+\delta e^{\beta_{0} \tau} \cdot e^{-\beta_{0} t} \leq \delta^{*} .
$$

Again (2.23) follows from (2.21) and (2.18).

Combining cases (i)-(iii), we have

$$
\frac{\partial w^{+}}{\partial t}-d \Delta w^{+}-f\left(w^{+}(x, t), w^{+}(x, t-\tau)\right) \geq 0, \quad x \in R, t \geq 0 .
$$

This completes the proof.

Let $\bar{\delta}_{0}=\min \left\{\frac{a^{-}}{2}, \frac{1-a^{+}}{2}, \delta_{0}\right\}$, and let $\zeta(\cdot) \in C^{\infty}(R)$ be a fixed function with the following properties:

$$
\begin{gathered}
\zeta(s)=0 \quad \text { if } s \leq 0 ; \quad \zeta(s)=1 \quad \text { if } s \geq 4 \\
0<\zeta^{\prime}(s)<1 ; \quad\left|\zeta^{\prime \prime}(s)\right| \leq 1 \quad \text { if } s \in(0,4)
\end{gathered}
$$

Then we have the following result.

Lemma 2.2 Assume that (H1) and (H2) hold. Then, for any $\delta \in\left(0, \bar{\delta}_{0}\right]$, there exist two positive numbers $\epsilon=\epsilon(\delta)$ and $C=C(\delta)$ such that, for every $\xi \in R$, the functions $v^{+}$and $v^{-}$defined by

$$
\begin{aligned}
& v^{+}(x, t):=(1+\delta)-\left[1-\left(a^{-}-2 \delta\right) e^{-\epsilon t}\right] \zeta(-\epsilon(x-\xi+C t)), \\
& v^{-}(x, t):=-\delta+\left[1-\left(1-a^{+}-2 \delta\right) e^{-\epsilon t}\right] \zeta(\epsilon(x-\xi-C t))
\end{aligned}
$$

are a super-solution and a sub-solution of (2.1) on $[0, \infty)$, respectively. 
Proof. By a translation, we can assume $\xi=0$. Given $\delta \in\left(0, \bar{\delta}_{0}\right]$, we define

$$
\begin{aligned}
& m_{1}=m_{1}(\delta)=\max \left\{\partial_{2} f(u, v) ;(u, v) \in[-\delta, 1-\delta]^{2}\right\} \geq 0, \\
& m_{2}=m_{2}(\delta)=\min \left\{\zeta^{\prime}(s) ; \frac{\delta}{2} \leq \zeta(s) \leq 1-\frac{\delta}{2}\right\}>0 .
\end{aligned}
$$

Then there exists an $\epsilon=\epsilon(\delta)>0$ such that

$$
\begin{gathered}
\left(1-a^{+}\right) e^{\epsilon \tau}<1, \\
-\min \left\{f_{0}(u) ; u \in\left[-\delta,-\frac{\delta}{2}\right]\right\}+\left(\epsilon+\tau m_{1} \epsilon+d \epsilon^{2}\right)<0,
\end{gathered}
$$

and

$$
-\min \left\{f_{0}(u) ; u \in\left[a^{+}+\frac{\delta}{2}, 1-\delta\right]\right\}+\left(\epsilon+\tau m_{1} \epsilon+d \epsilon^{2}\right)<0 .
$$

We further choose $C=C(\delta)>0$ such that

$$
-C \epsilon a^{+} m_{2}+\max \left\{\left|f_{0}(u)\right| ; u \in[-\delta, 1-\delta]\right\}+\left(\epsilon+\tau m_{1} \epsilon+d \epsilon^{2}\right)<0 .
$$

By a direct computation and (2.26), it follows that for all $t \geq-\tau$,

$$
\begin{aligned}
\frac{\partial v^{-}(x, t)}{\partial t}= & -C \epsilon\left[1-\left(1-a^{+}-2 \delta\right) e^{-\epsilon t}\right] \zeta^{\prime}(\epsilon(x-C t)) \\
& +\epsilon\left(1-a^{+}-2 \delta\right) \cdot \zeta(\epsilon(x-C t)) e^{-\epsilon t} \\
\leq & -C \epsilon\left[1-\left(1-a^{+}\right) e^{\epsilon \tau}\right] \zeta^{\prime}(\epsilon(x-C t))+\epsilon\left(1-a^{+}\right) \zeta(\epsilon(x-C t)) e^{\epsilon \tau} \\
\leq & \epsilon .
\end{aligned}
$$

It is easy to see that $v^{-}(x, t) \in[-\delta, 1-\delta]$, for all $x \in R$ and $t \geq-\tau$. Therefore we have that for all $t \geq 0$,

$$
\begin{aligned}
f\left(v^{-}(x, t), v^{-}(x, t-\tau)\right)= & f_{0}\left(v^{-}(x, t)\right)+\left[f\left(v^{-}(x, t), v^{-}(x, t-\tau)\right)\right. \\
& \left.-f\left(v^{-}(x, t), v^{-}(x, t)\right)\right] \\
= & f_{0}\left(v^{-}(x, t)\right)+\partial_{2} f\left(v^{-}(x, t), v^{*}(x, t)\right)\left(v^{-}(x, t-\tau)\right. \\
& \left.-v^{-}(x, t)\right) \\
= & f_{0}\left(v^{-}(x, t)\right)+\partial_{2} f_{2}\left(v^{-}(x, t), v^{*}(x, t)\right) \frac{\partial v^{-}\left(x, t^{*}\right)}{\partial t} . \\
& ((t-\tau)-t) \\
\geq & f_{0}\left(v^{-}(x, t)\right)-\tau \epsilon m_{1},
\end{aligned}
$$


where $v^{*}(x, t)$ is between $v^{-}(x, t)$ and $v^{-}(x, t-\tau), t^{*} \in[t-\tau, t]$ and hence $t^{*} \geq-\tau$. It then follows that

$$
\begin{aligned}
& \frac{\partial v^{-}(x, t)}{\partial t}-d \Delta v^{-}(x, t)-f\left(v^{-}(x, t), v^{-}(x, t-\tau)\right) \\
& \quad=-C \epsilon\left[1-\left(1-a^{+}-2 \delta\right) e^{-\epsilon t}\right] \zeta^{\prime}\left(\epsilon(x-C t)+\epsilon\left(1-a^{+}-2 \delta\right) \zeta(\epsilon(x-C t)) .\right. \\
& e^{-\epsilon t}-d\left[1-\left(1-a^{+}-2 \delta\right) e^{-\epsilon t}\right] \zeta^{\prime \prime}(\epsilon(x-C t)) \epsilon^{2}-f\left(v^{-}(x, t), v^{-}(x, t-\tau)\right) \\
& \leq-C \epsilon a^{+} \zeta^{\prime}(\epsilon(x-C t))+\epsilon+d \epsilon^{2}-\left(f_{0}\left(v^{-}(x, t)-\tau \epsilon m_{1}\right)\right. \\
& \quad=-C \epsilon a^{+} \zeta^{\prime}(\epsilon(x-C t))-f_{0}\left(v^{-}(x, t)\right)+\left(\epsilon+\tau \epsilon m_{1}+d \epsilon^{2}\right) .
\end{aligned}
$$

We distinguish among three cases:

Case(i) $\zeta(\epsilon(x-C t))<\frac{\delta}{2}$ : Clearly, $v^{-}(x, t) \in\left[-\delta,-\frac{\delta}{2}\right]$ for all $x \in R$ and $t \geq 0$. By (2.32) and (2.27), it follows that

$$
\begin{gathered}
\frac{\partial v^{-}}{\partial t}-d_{0} \Delta v^{-}-f\left(v^{-}(x, t), v^{-}(x, t-\tau)\right) \\
\leq-\min \left\{f_{0}(u) ; u \in\left[-\delta,-\frac{\delta}{2}\right]\right\} \\
+\left(\epsilon+\tau \epsilon m_{1}+d \epsilon^{2}\right)<0 .
\end{gathered}
$$

Case (ii) $\zeta(\epsilon(x-C t))>1-\frac{\delta}{2}$ : It then follows that

$$
\begin{aligned}
1-\delta \geq v^{-}(x, t) & \geq-\delta+\left(1-\frac{\delta}{2}\right)\left[1-\left(1-a^{+}-2 \delta\right)\right] \\
& =a^{+}+\frac{\delta}{2}\left[2-\left(2 \delta+a^{+}\right)\right] \\
& \geq a^{+}+\frac{\delta}{2} .
\end{aligned}
$$

Therefore, by (2.32) and (2.28), we have

$$
\begin{gathered}
\frac{\partial v^{-}}{\partial t}-d \Delta v^{-}-f\left(v^{-}(x, t), v^{-}(x, t-\tau)\right) \\
\leq-\min \left\{f_{0}(u) ; u \in\left[a^{+}+\frac{\delta}{2}, 1-\delta\right]\right\} \\
+\left(\epsilon+\tau \epsilon m_{1}+d \epsilon^{2}\right)<0 .
\end{gathered}
$$

Case (iii) $\zeta(\epsilon(x-C t)) \in\left[\frac{\delta}{2}, 1-\frac{\delta}{2}\right]$ : By (2.32) and (2.29), we have

$$
\begin{aligned}
\frac{\partial v^{-}}{\partial t}-d_{0} \Delta v^{-}-f\left(v^{-}(x, t), v^{-}(x, t-\tau)\right) \leq & -C \epsilon a^{+} m_{2}+\max \left\{\left|f_{0}(u)\right|\right. \\
& u \in[-\delta, 1-\delta]\} \\
& +\left(\epsilon+\tau \epsilon m_{1}+d \epsilon^{2}\right)<0 .
\end{aligned}
$$


Combining cases (i)-(iii), we have

$$
\frac{\partial v^{-}}{\partial t}-d \Delta v^{-}-f\left(v^{-}(x, t), v^{-}(x, t-\tau)\right) \leq 0, \quad x \in R, \quad t \geq 0 .
$$

Then $v^{-}(x, t)$ is a sub-solution of $(2.1)$ on $[0, \infty)$. In a similar way, we can prove $v^{+}(x, t)$ is a super-solution of $(2.1)$ on $[0, \infty)$.

This completes the proof.

Remark 2.2 It is easy to see $v^{+}(x, t)$ and $v^{-}(x, t)$ in Lemma 2.2 have the following properties:

$(P 1) v^{+}(x, t)=1+\delta$ for all $x \geq \xi+C \tau$ and $t \in[-\tau, 0] ; v^{+}(x, t) \geq a^{-}-\delta$ for all $x \in R$ and $t \in[-\tau, 0] ; v^{+}(x, t) \leq \delta+(a-2 \delta) e^{-\epsilon t}$ for all $x \leq \xi-C t-4 \epsilon^{-1}$ and $t \in[0, \infty)$.

(P2) $v^{-}(x, t)=-\delta$ for all $x \leq \xi-C \tau$ and $t \in[-\tau, 0] ; v^{-}(x, t) \leq a^{+}+\delta$ for all $x \in R$ and $t \in[-\tau, 0] ; v^{-}(x, t) \geq 1-\delta-\left(1-a^{+}-2 \delta\right) e^{-\epsilon t}$ for all $x \geq \xi+C t+4 \epsilon^{-1}$ and $t \in[0, \infty)$.

Lemma 2.3 For any traveling wave solution $U(x-c t)$ of (2.1) with $0 \leq$ $U(\xi) \leq 1, \xi \in R$, there holds $\lim _{\xi \rightarrow \pm \infty} U^{\prime}(\xi)=0$.

Proof. We only prove $\lim _{\xi \rightarrow+\infty} U^{\prime}(\xi)=0$ since $\lim _{\xi \rightarrow-\infty} U^{\prime}(\xi)=0$ follows by the transformation $V(\xi)=U(-\xi)$. By Theorem 2.1, $U(\cdot) \in C^{2}(R)$ and $U(\xi)$ satisfies $(2.20)$. In the case where $c=0,(2.20)$ implies that $U^{\prime \prime}(\cdot)$ is bounded on $R$ and hence $U^{\prime}(\cdot)$ is uniformly continuous on $R$. Then, by (2.17) and Barbalat lemma (see, e.g., [5]), $\lim _{\xi \rightarrow+\infty} U^{\prime}(\xi)=0$. In the case where $c \neq 0$, we first claim that $\lim _{\xi \rightarrow+\infty} U^{\prime}(\xi)$ exists. Assume that, by contradiction, $\liminf _{\xi \rightarrow+\infty} U^{\prime}(\xi)<\lim \sup _{\xi \rightarrow+\infty} U^{\prime}(\xi)$. Then, by fluctuation lemma (see [6]), there are two sequences $\left\{\xi_{n}\right\}_{n=1}^{\infty}$ and $\left\{s_{n}\right\}_{n=1}^{\infty}$ with $\xi_{n} \rightarrow+\infty$ and $s_{n} \rightarrow+\infty$ as $n \rightarrow+\infty$ such that

$$
\lim _{n \rightarrow+\infty} U^{\prime}\left(\xi_{n}\right)=\limsup _{\xi \rightarrow+\infty} U^{\prime}(\xi), \quad U^{\prime \prime}\left(\xi_{n}\right)=0, \quad n \geq 1
$$

and

$$
\lim _{n \rightarrow+\infty} U^{\prime}\left(s_{n}\right)=\liminf _{\xi \rightarrow+\infty} U^{\prime}(\xi), \quad U^{\prime \prime}\left(s_{n}\right)=0, \quad n \geq 1 .
$$

Since $\lim _{\xi \rightarrow+\infty} U(\xi)=1$ and $f(1,1)=0$, letting $n \rightarrow+\infty$ in (2.20) with $\xi$ replaced by $\xi_{n}$ and $s_{n}$, respectively, we get that $\lim \sup _{\xi \rightarrow+\infty} U^{\prime}(\xi)=$ 
$0=\liminf _{\xi \rightarrow+\infty} U^{\prime}(\xi)$, which is a contradiction to our assumption. Let $\lim _{\xi \rightarrow+\infty} U^{\prime}(\xi)=L$. Since $U(n+1)-U(n)=U^{\prime}\left(t_{n}\right)$, where $t_{n} \in[n, n+1]$, letting $n \rightarrow \infty$, we have $L=1-1=0$.

This completes the proof.

\section{$3 \quad$ Stability and uniqueness of traveling waves}

In this section we will discuss the global asymptotic stability with shift and stability of monotone traveling wave and uniqueness of traveling waves. To prove our main results, we need the following two lemmas.

Let $U(x-c t)$ be a monotone traveling wave solution of $(2.1)$. In view of Lemma 2.1, we define the following two functions

$$
\begin{gathered}
w^{ \pm}(x, t, \eta, \delta)=U\left(x-c t+\eta \pm \sigma_{0} \delta\left(1-e^{-\beta_{0} t}\right)\right) \pm \delta e^{-\beta_{0} t} \\
x \in R, \quad t \in[-\tau, \infty), \quad \eta \in R, \text { and } \delta \in[0, \infty)
\end{gathered}
$$

where $\sigma_{0}, \beta_{0}$ are as in Lemma 2.1. By the proof of Lemma 2.1 we can choose $\beta_{0}>0$ as small as we wish. Then we assume that $\beta_{0}$ has been chosen such that $3 e^{\beta_{0} \tau}<4$ throughout this section.

Lemma 3.1 Let $U(x-c t)$ be a monotone traveling wave solution of (2.1). Then there exists a positive number $\epsilon^{*}$ such that, if $u(x, t)$ is a solution of (2.1) on $[0, \infty)$ with $0 \leq u(x, t) \leq 1$ for $x \in R$ and $t \in[0, \infty)$, and for some $\xi \in R, h>0,0<\delta<\min \left(\bar{\delta}, \frac{1}{\sigma_{0}}\right)$ and $T \geq 0$, there holds

$$
\begin{gathered}
w_{0}^{-}(x,-c T+\xi, \delta)(s) \leq u_{T}(x)(s) \leq w_{0}^{+}(x,-c T+\xi+h, \delta)(s), \\
s \in[-\tau, 0], x \in R,
\end{gathered}
$$

then for every $t \geq T+\tau+1$, there exist $\hat{\xi}(t), \hat{\delta}(t)$ and $\hat{h}(t)$ such that

$$
\begin{gathered}
w_{0}^{-}(x,-c t+\hat{\xi}(t), \hat{\delta}(t))(s) \leq u_{t}(x)(s) \leq w_{0}^{+}(x,-c t+\hat{\xi}(t)+\hat{h}(t), \hat{\delta}(t))(s), \\
s \in[-\tau, 0], \quad x \in R,
\end{gathered}
$$

with $\hat{\xi}(t), \hat{\delta}(t)$ and $\hat{h}(t)$ satisfying

$$
\begin{gathered}
\hat{\xi}(t) \in\left[\xi-\sigma_{0} \delta-2 \sigma_{0}\left(\delta+\epsilon^{*} \min (h, 1)\right) e^{\beta_{0} \tau}, \xi+h+\sigma_{0} \delta\right], \\
\hat{\delta}(t)=\left(\delta e^{-\beta_{0}}+\epsilon^{*} \min (1, h)\right) e^{-\beta_{0}(t-(T+\tau+1))} \\
\hat{h}(t) \in\left[0, h+\left(3 e^{\beta_{0} \tau}-4\right) \sigma_{0} \epsilon^{*} \min (h, 1)+3 e^{\beta_{0} \tau} \sigma_{0} \delta\right] .
\end{gathered}
$$


Proof. By Lemma 2.1, $w^{+}(x, t,-c T+\xi+h, \delta)$ and $w^{-}(x, t,-c T+\xi, \delta)$ are super- and sub-solutions of (2.1), respectively. Clearly, $v(x, t)=u(x, T+t)$, $t \geq 0$, is also a solution of $(2.1)$ with $v_{0}(x)(s)=u_{T}(x)(s), s \in[-\tau, 0], x \in R$. Then, by Theorem 2.1, there holds

$$
\begin{gathered}
w^{-}(x, t,-c T+\xi, \delta) \leq u(x, T+t) \leq w^{+}(x, t,-c T+\xi+h, \delta), \\
x \in R, t \geq 0 .
\end{gathered}
$$

That is,

$$
\begin{aligned}
U\left(x-c(T+t)+\xi-\sigma_{0} \delta\left(1-e^{-\beta_{0} t}\right)\right)-\delta e^{-\beta_{0} t} \\
\leq u(x, T+t) \\
\leq U\left(x-c(T+t)+\xi+h+\sigma_{0} \delta\left(1-e^{-\beta_{0} t}\right)\right) \\
\quad+\delta e^{-\beta_{0} t}, \quad x \in R, t \geq 0 .
\end{aligned}
$$

Let $z=c T-\xi$. Again by Theorem 2.1, we have that for any $J \geq 0$, all $x \in R$ with $|x-z| \leq J$ and all $t>0$,

$$
\begin{aligned}
& u(x, T+t)-w^{-}(x, t,-c T+\xi, \delta) \\
& \quad \geq \theta(J, t) \int_{z}^{z+1}\left(u(y, T)-w^{-}(y, 0,-c T+\xi, \delta)\right) d y \\
& \quad=\theta(J, t) \int_{z}^{z+1}[u(y, T)-(U(y-c T+\xi)-\delta)] d y \\
& \quad=\theta(J, t)\left[\int_{z}^{z+1}(u(y, T)-U(y-c T+\xi)) d y+\delta\right] .
\end{aligned}
$$

By Lemma 2.3, $\lim _{|\eta| \rightarrow \infty} U^{\prime}(\eta)=0$. Then we can fix a positive number $M$ such that $U^{\prime}(\eta) \leq \frac{1}{2 \sigma_{0}}$ for all $|\eta| \geq M$. Let $J=M+|c|(1+\tau)+1$, $\bar{h}=\min (1, h)$, and

$$
\epsilon_{1}=\frac{1}{2} \min \left\{U^{\prime}(x) ;|x| \leq 2\right\}>0
$$

Then

$\int_{z}^{z+1}[U(y-c T+\xi+\bar{h})-U(y-c T+\xi)] d y=\int_{0}^{1}(U(y+\bar{h})-U(y)) d y \geq 2 \epsilon_{1} \bar{h}$,

and hence, at least one of the following is true:

(i) $\int_{z}^{z+1}[u(y, T)-U(y-c T+\xi)] d y \geq \epsilon_{1} \bar{h}$;

(ii) $\int_{z}^{z+1}[U(y-c T+\xi+\bar{h})-u(y, T)] d y \geq \epsilon_{1} \bar{h}$. 
In what follows, we consider only the case (i). The case (ii) is similar and thus omitted. For any $s \in[-\tau, 0],|x-z| \leq J$, letting $t=1+\tau+s \geq 1$ in (3.3), we have

$$
\begin{aligned}
u(x, T+1+\tau+s) \geq & U\left(x-z-c(1+\tau+s)-\sigma_{0} \delta\left(1-e^{-\beta_{0}(1+\tau+s)}\right)\right) \\
& -\delta e^{-\beta_{0}(1+\tau+s)}+\theta_{0}(J) \epsilon_{1} \bar{h},
\end{aligned}
$$

where $\theta_{0}(J)=\min _{s \in[-\tau, 0]} \theta(J, 1+\tau+s)$. Let

$$
\begin{aligned}
J_{1} & =J+|c|(1+\tau)+3, \\
\epsilon^{*} & =\min \left\{\min _{|x| \leq J_{1}} \frac{\theta_{0}(J) \epsilon_{1}}{2 \sigma_{0} U^{\prime}(x)}, \frac{1}{3 \sigma_{0}}\right\} .
\end{aligned}
$$

By the mean value theorem, it then follows that for all $|x-z| \leq J, s \in[-\tau, 0]$,

$$
\begin{aligned}
& U\left(x-z-c(1+\tau+s)+2 \sigma_{0} \epsilon^{*} \bar{h}-\sigma_{0} \delta\left(1-e^{-\beta_{0}(1+\tau+s)}\right)\right) \\
& -U\left(x-z-c(1+\tau+s)-\sigma_{0} \delta\left(1-e^{-\beta_{0}(1+\tau+s)}\right)\right) \\
& =U^{\prime}\left(\eta_{1}\right) 2 \sigma_{0} \epsilon^{*} \bar{h} \leq \theta_{0}(J) \epsilon_{1} \bar{h}
\end{aligned}
$$

and hence,

$$
\begin{aligned}
u(x, T+1+\tau+s) \geq & U\left(x-c(T+1+\tau+s)+\xi+2 \sigma_{0} \epsilon^{*} \bar{h}\right. \\
& \left.-\sigma_{0} \delta\left(1-e^{-\beta_{0}(1+\tau+s)}\right)\right)-\delta e^{-\beta_{0}(1+\tau+s)} .
\end{aligned}
$$

By the choice of $M$ and $J$ and by the mean value theorem, it then follows that for all $|x-z| \geq J, s \in[-\tau, 0]$,

$$
\begin{aligned}
& U\left(x-c(T+1+\tau+s)+\xi-\sigma_{0} \delta\left(1-e^{-\beta_{0}(1+\tau+s)}\right)\right) \\
& -U\left(x-c(T+1+\tau+s)+2 \sigma_{0} \epsilon^{*} \bar{h}+\xi-\sigma_{0} \delta\left(1-e^{-\beta_{0}(1+\tau+s)}\right)\right) \\
& =U^{\prime}\left(\eta_{2}\right)\left(-2 \sigma_{0} \epsilon^{*} \bar{h}\right) \geq-\epsilon^{*} \bar{h} .
\end{aligned}
$$

That is, for all $|x-z| \geq J, s \in[-\tau, 0]$,

$$
\begin{aligned}
& U\left(x-c(T+1+\tau+s)+\xi-\sigma_{0} \delta\left(1-e^{-\beta_{0}(1+\tau+s)}\right)\right) \\
& \geq U\left(x-c(T+1+\tau+s)+\xi+2 \sigma_{0} \epsilon^{*} \bar{h}\right. \\
& \left.-\sigma_{0} \delta\left(1-e^{-\beta_{0}(1+\tau+s)}\right)\right)-\epsilon^{*} \bar{h}
\end{aligned}
$$

and hence, by (3.2) with $t=1+\tau+s$, we have

$$
\begin{aligned}
u(x, T+1+\tau+s) \geq & U\left(x-c(T+1+\tau+s)+\xi+2 \sigma_{0} \epsilon^{*} \bar{h}\right. \\
& \left.-\sigma_{0} \delta\left(1-e^{-\beta_{0}(1+\tau+s)}\right)\right) \\
& -\epsilon^{*} \bar{h}-\delta e^{-\beta_{0}(1+\tau+s)},
\end{aligned}
$$


for all $|x-z| \geq J, \quad s \in[-\tau, 0]$. By (3.6) and (3.9), it follows that for all $x \in R, x \in[-\tau, 0]$,

$$
\begin{aligned}
u(x, T+1+\tau+s) \geq & U\left(x-c(T+1+\tau+s)+\xi+2 \sigma_{0} \epsilon^{*} \bar{h}\right. \\
& \left.-\sigma_{0} \delta\left(1-e^{-\beta_{0}(1+\tau+s)}\right)\right) \\
& -\delta e^{-\beta_{0}(1+\tau+s)}-\epsilon^{*} \bar{h},
\end{aligned}
$$

and hence,

$$
\begin{aligned}
u_{T+1+\tau}(x)(s) \geq & U\left(x-c s+\left[-c(T+1+\tau)+\xi+2 \sigma_{0} \epsilon^{*} \bar{h}\right.\right. \\
& -\sigma_{0} \delta\left(1-e^{-\beta_{0}(1+\tau+s)}\right)+\sigma_{0}\left(\delta e^{-\beta_{0}}+\epsilon^{*} \bar{h}\right)\left(1-e^{-\beta_{0} s}\right) \\
& \left.\left.-\sigma_{0}\left(\delta e^{-\beta_{0}}+\epsilon^{*} \bar{h}\right)\left(1-e^{-\beta_{0} s}\right)\right]\right)-\left(\delta e^{-\beta_{0}}+\epsilon^{*} \bar{h}\right) e^{-\beta_{0} s} \\
\geq & U\left(x-c s+\left(-c(T+1+\tau)+\xi+2 \sigma_{0} \epsilon^{*} \bar{h}+\bar{\xi}\right)\right. \\
& \left.-\sigma_{0}\left(\delta e^{-\beta_{0}}+\epsilon^{*} \bar{h}\right)\left(1-e^{-\beta_{0} s}\right)\right) \\
& -\left(\delta e^{-\beta_{0}}+\epsilon^{*} \bar{h}\right) e^{-\beta_{0} s}
\end{aligned}
$$

where

$$
\bar{\xi}=-\sigma_{0} \delta+\sigma_{0}\left(\delta e^{-\beta_{0}}+\epsilon^{*} \bar{h}\right)\left(1-e^{\beta_{0} \tau}\right)
$$

Then

$$
u_{T+1+\tau}(x)(s) \geq w_{0}^{-}\left(x, \eta, \delta e^{-\beta_{0}}+\epsilon^{*} \bar{h}\right)(s), \quad x \in R, s \in[-\tau, 0],
$$

where $\eta=-c(T+1+\tau)+\xi+2 \sigma_{0} \epsilon^{*} \bar{h}+\bar{\xi}$. Again, by Theorem 2.1,

$$
u_{T+1+\tau+t^{\prime}}(x)(s) \geq w_{t^{\prime}}^{-}\left(x, \eta, \delta e^{-\beta_{0}}+\epsilon^{*} \bar{h}\right)(s), \quad \text { for all } t^{\prime} \geq 0, s \in[-\tau, 0] .
$$

Then for any $t \geq T+1+\tau$, setting $t^{\prime}=t-(T+1+\tau)$ in (3.14), we have

$$
\begin{aligned}
& u_{t}(x)(s) \geq w_{t-(T+1+\tau)}^{-}\left(x, \eta, \delta e^{-\beta_{0}}+\epsilon^{*} \bar{h}\right)(s) \\
&= U\left(x-c s-c t+c(T+1+\tau)+\eta-\sigma_{0}\left(\delta e^{-\beta_{0}}+\epsilon^{*} \bar{h}\right) .\right. \\
&\left.\left(1-e^{-\beta_{0}(t-(T+1+\tau))} \cdot e^{-\beta_{0} s}\right)\right) \\
&-\left(\delta e^{-\beta_{0}}+\epsilon^{*} \bar{h}\right) e^{-\beta_{0}(t-(T+1+\tau))} \cdot e^{-\beta_{0} s} \\
& \geq U\left(x-c s-c t+c(T+1+\tau)+\eta-\sigma_{0}\left(\delta e^{-\beta_{0}}+\epsilon^{*} \bar{h}\right) \cdot 1\right. \\
&\left.+\sigma_{0} \hat{\delta}(t)\left(1-e^{-\beta_{0} s}\right)-\sigma_{0} \hat{\delta}(t)\left(1-e^{-\beta_{0} s}\right)\right)-\hat{\delta}(t) e^{-\beta_{0} s} \\
& \geq \quad U\left(x-c s-c t+c(T+1+\tau)+\eta-\sigma_{0}\left(\delta e^{-\beta_{0}}+\epsilon^{*} \bar{h}\right)\right. \\
&\left.\left.+\sigma_{0} \hat{\delta}(t)\left(1-e^{\beta_{0} \tau}\right)\right]-\sigma_{0} \hat{\delta}(t)\left(1-e^{-\beta_{0} s}\right)\right)-\hat{\delta}(t) \cdot e^{-\beta_{0} s},(3
\end{aligned}
$$


where $\hat{\delta}(t)=\left(\delta e^{-\beta_{0}}+\epsilon^{*} \bar{h}\right) \cdot e^{-\beta_{0}(t-(T+1+\tau))}$. By the monotonicity of $U(\cdot)$, the choice of $\eta$ and (3.12), it then follows that

$$
u_{t}(x)(s) \geq w_{0}^{-}(x,-c t+\hat{\xi}(t), \hat{\delta}(t))(s), \quad s \in[-\tau, 0], x \in R,
$$

where

$$
\begin{aligned}
\hat{\xi}(t)= & \xi+2 \sigma_{0} \epsilon^{*} \bar{h}+\left[-\sigma_{0} \delta+\sigma_{0}\left(\delta e^{-\beta_{0}}+\epsilon^{*} \bar{h}\right)\left(1-e^{\beta_{0} \tau}\right)\right] \\
& -\sigma_{0}\left(\delta e^{-\beta_{0}}+\epsilon^{*} \bar{h}\right)+\sigma_{0} \hat{\delta}(t)\left(1-e^{\beta_{0} \tau}\right) \\
= & \xi+2 \sigma_{0} \epsilon^{*} \bar{h}-\sigma_{0} \delta-\sigma_{0}\left(\delta e^{-\beta_{0}}+\epsilon^{*} \bar{h}\right) e^{\beta_{0} \tau} \\
& +\sigma_{0} \hat{\delta}(t)-\sigma_{0} \hat{\delta}(t) e^{\beta_{0} \tau} .
\end{aligned}
$$

Therefore it follows that

$$
\begin{aligned}
\hat{\xi}(t) & \geq \xi-\sigma_{0} \delta-\sigma_{0}\left(\delta e^{-\beta_{0}}+\epsilon^{*} \bar{h}\right) e^{\beta_{0} \tau}-\sigma_{0} \hat{\delta}(t) e^{\beta_{0} \tau} \\
& \geq \xi-\sigma_{0} \delta-2 \sigma_{0}\left(\delta e^{-\beta_{0}}+\epsilon^{*} \bar{h}\right) e^{\beta_{0} \tau} \\
& \geq \xi-\sigma_{0} \delta-2 \sigma_{0}\left(\delta+\epsilon^{*} \bar{h}\right) e^{\beta_{0} \tau}
\end{aligned}
$$

and, by the choice of $\epsilon^{*}$,

$$
\begin{aligned}
\hat{\xi}(t) & \leq \xi+2 \sigma_{0} \epsilon^{*} \bar{h}+\sigma_{0}\left(\delta e^{-\beta_{0}}+\epsilon^{*} \bar{h}\right) \\
& \leq \xi+3 \sigma_{0} \epsilon^{*} \bar{h}+\sigma_{0} \delta \\
& \leq \xi+h+\sigma_{0} \delta
\end{aligned}
$$

For any $t \geq T$, by the inequality of the righthand side of (3.2), we have

$$
u(x, t) \leq U\left(x-c t+\xi+h+\sigma_{0} \delta\left(1-e^{-\beta_{0}(t-T)}\right)+\delta e^{-\beta_{0}(t-T)} .\right.
$$

It then follows that, for all $t \geq T+1+\tau$,

$$
\begin{aligned}
u_{t}(x)(s)= & u(x, t+s) \leq U(x-c(t+s)+\xi+h \\
& +\sigma_{0} \delta\left(1-e^{-\beta_{0}(t+s-T)}\right)+\delta e^{-\beta_{0}(t+s-T)}, \\
& s \in[-\tau, 0], x \in R .
\end{aligned}
$$

Therefore for all $t \geq T+1+\tau$,

$$
\begin{aligned}
u_{t}(x)(s) \leq & U\left(x-c s-c t+\xi+h+\sigma_{0} \delta\left(1-e^{-\beta_{0}(t+s-T)}\right)+\hat{\delta}(t) e^{-\beta_{0} s}\right. \\
= & U\left(x-c s-c t+\xi+h+\sigma_{0} \delta\left(1-e^{-\beta_{0}(t-T)} \cdot e^{-\beta_{0} s}\right)\right. \\
& \left.-\sigma_{0} \hat{\delta}(t)\left(1-e^{-\beta_{0} s}\right)+\sigma_{0} \hat{\delta}(t)\left(1-e^{-\beta_{0} s}\right)\right)+\hat{\delta}(t) e^{-\beta_{0} s} \\
\leq & U\left(x-c s-c t+\xi+h+\sigma_{0} \delta-\sigma_{0} \hat{\delta}(t)\left(1-e^{\beta_{0} \tau}\right)\right. \\
& \left.+\sigma_{0} \hat{\delta}(t)\left(1-e^{-\beta_{0} s}\right)\right)+\hat{\delta}(t) e^{-\beta_{0} s}, \quad s \in[-\tau, 0], x \in R .
\end{aligned}
$$


It then follows that for all $t \geq T+1+\tau$,

$$
u_{t}(x)(s) \leq w_{0}^{+}(x,-c t+\hat{\xi}(t)+\hat{h}(t), \hat{\delta}(t))(s), \quad s \in[-\tau, 0], x \in R,
$$

where

$$
\begin{aligned}
\hat{h}(t)= & \xi+h+\sigma_{0} \delta-\sigma_{0} \hat{\delta}(t)\left(1-e^{\beta_{0} \tau}\right)-\hat{\xi}(t) \\
= & h-2 \sigma_{0} \epsilon^{*} \bar{h}+2 \sigma_{0} \delta+2 \sigma_{0} \hat{\delta}(t)\left(e^{\beta_{0} \tau}-1\right) \\
& +\sigma_{0}\left(\delta e^{-\beta_{0}}+\epsilon^{*} \bar{h}\right) e^{\beta_{0} \tau} .
\end{aligned}
$$

Notice that we have used the expression of $\hat{\xi}(t)$ in getting the second equality of (3.23). By the choice of $\epsilon^{*}$, we have $h-2 \sigma_{0} \epsilon^{*} \bar{h} \geq h-2 \sigma_{0} \epsilon^{*} h=(1-$ $\left.2 \sigma_{0} \epsilon^{*}\right) h>0$, and hence,

$$
\begin{aligned}
0< & \hat{h}(t) \leq h-2 \sigma_{0} \epsilon^{*} \bar{h}+2 \sigma_{0} \delta+2 \sigma_{0}\left(\delta e^{-\beta_{0}}+\epsilon^{*} \bar{h}\right) \cdot\left(e^{\beta_{0} \tau}-1\right) \\
& +\sigma_{0}\left(\delta e^{-\beta_{0}}+\epsilon^{*} \bar{h}\right) e^{\beta_{0} \tau} \\
\leq & h-2 \sigma_{0} \epsilon^{*} \bar{h}+2 \sigma_{0} \delta+\sigma_{0}\left(\delta e^{-\beta_{0}}+\epsilon^{*} \bar{h}\right) \cdot\left(3 e^{\beta_{0} \tau}-2\right) \\
\leq & h-2 \sigma_{0} \epsilon^{*} \bar{h}+2 \sigma_{0} \delta+\sigma_{0}\left(\delta+\epsilon^{*} \bar{h}\right)\left(3 e^{\beta_{0} \tau}-2\right) \\
= & h+\left(3 e^{\beta_{0} \tau}-4\right) \sigma_{0} \epsilon^{*} \bar{h}+3 e^{\beta_{0} \tau} \cdot \sigma_{0} \delta
\end{aligned}
$$

Combining (3.16) and (3.22), now we complete the proof.

Lemma 3.2 Let $U(x-c t)$ be a monotone traveling wave solution of (2.1), and let $\varphi \in[0,1]_{C}$ be such that

$$
\liminf _{x \rightarrow \infty} \min _{s \in[-\tau, 0]} \varphi(x, s)>a^{+}, \quad \limsup _{x \rightarrow-\infty} \max _{s \in[-\tau, 0]} \varphi(x, s)<a^{-} .
$$

Then, for any $\delta>0$, there exist $T=T(\varphi, \delta)>0, \xi=\xi(\varphi, \delta) \in R$ and $h=h(\varphi, \delta)>0$ such that

$$
\begin{gathered}
w_{0}^{-}(x,-c T+\xi, \delta)(s) \leq u_{T}(x, \varphi)(s) \leq w_{0}^{+}(x,-c T+\xi+h, \delta)(s), \\
s \in[-\tau, 0], x \in R .
\end{gathered}
$$

Proof. By Theorem 2.1, $u(x, t, \varphi)$ exists globally on $[0, \infty)$ and $0 \leq u(x, t, \varphi) \leq$ $1, x \in R, t \geq 0$. For any $\delta>0$, we choose a $0<\delta_{1}=\delta_{1}(\delta, \varphi)<\min \left(\delta, \bar{\delta}_{0}\right)$ such that

$$
\begin{aligned}
& \liminf _{x \rightarrow \infty} \min _{s \in[-\tau, 0]} \varphi(x, s)>a^{+}+\delta_{1}, \\
& \limsup _{x \rightarrow-\infty} \max _{s \in[-\tau, 0]} \varphi(x, s)<a^{-}-\delta_{1} .
\end{aligned}
$$


Then there exists $M=M\left(\varphi, \delta_{1}\right)>0$ such that

$$
\begin{aligned}
& \varphi(x, s) \leq a^{-}-\delta_{1}, \quad \text { for all } x \leq-M \text { and } s \in[-\tau, 0], \\
& \varphi(x, s) \geq a^{+}+\delta_{1}, \quad \text { for all } x \geq M \text { and } s \in[-\tau, 0] .
\end{aligned}
$$

Let $\epsilon=\epsilon\left(\delta_{1}\right)$ and $C=C\left(\delta_{1}\right)$ be defined in Lemma 2.2 with $\delta$ replaced by $\delta_{1}$. Define $\xi^{+}=-M-C \tau$ and $\xi^{-}=M+C \tau$, and let $v^{ \pm}(x, t)$ be defined in Lemma 2.2 with $\xi=\xi^{ \pm}$, respectively. By (3.25) and Remark 2.2, it follows that for all $s \in[-\tau, 0]$,

$$
\begin{aligned}
& \varphi(x, s) \leq a^{-}-\delta_{1} \leq v^{+}(x, s) \quad \text { for } x \leq-M \\
& \varphi(x, s) \leq 1<1+\delta_{1}=v^{+}(x, s) \quad \text { for } x \geq \xi^{+}+C \tau=-M
\end{aligned}
$$

and

$$
\begin{array}{ll}
\varphi(x, s) \geq a^{+}+\delta_{1} \geq v^{-}(x, s) & \text { for } x \geq M \\
\varphi(x, s) \geq 0>-\delta_{1}=v^{-}(x, s) & \text { for } x \leq \xi^{-}-C \tau=M .
\end{array}
$$

Then we have

$$
v^{-}(x, s) \leq \varphi(x, s) \leq v^{+}(x, s), \quad x \in R, s \in[-\tau, 0] .
$$

By Lemma 2.2 and Theorem 2.1, it follows that

$$
v^{-}(x, t) \leq u(x, t, \varphi) \leq v^{+}(x, t), \quad x \in R, t \geq 0 .
$$

Since $\delta_{1}<\delta$, we choose a sufficiently large positive number $T>\tau$ such that, for all $t \geq T-\tau$,

$$
\delta_{1}+\left(a^{-}-2 \delta_{1}\right) e^{-\epsilon t}<\delta \text { and } 1-\delta_{1}-\left(1-a^{+}-2 \delta_{1}\right) e^{-\epsilon t}>1-\delta,
$$

and hence, again by Remark 2.2,

$$
u(x, t, \varphi) \leq v^{+}(x, t)<\delta, \quad \text { for } x \leq \xi^{+}-C t-4 \epsilon^{-1},
$$

and

$$
u(x, t, \varphi) \geq v^{-}(x, t)>1-\delta, \quad \text { for } x \geq \xi^{-}+C t+4 \epsilon^{-1} .
$$

Let $x^{-}=\xi^{+}-C T-4 \epsilon^{-1}$ and $x^{+}=\xi^{-}+C T+4 \epsilon^{-1}$. By (3.28) and (3.29), it follows that for any $t \in[T-\tau, T]$,

$$
\begin{aligned}
& u(x, t, \varphi)<\delta, \quad \text { for } x \leq x^{-} \\
& u(x, t, \varphi)>1-\delta, \quad \text { for } x \geq x^{+} .
\end{aligned}
$$


By (2.17), there exists a sufficiently large positive number $H$ such that $\frac{H}{2}>$ $x^{+},-\frac{H}{2}<x^{-}$and

$$
U(x)+\delta>1 \quad \text { for all } x \geq \frac{H}{2}, \quad \text { and } U(x)-\delta<0 \quad \text { for all } x \leq-\frac{H}{2} .
$$

Combining (3.30), (3.31) and the fact that $0 \leq U(x) \leq 1$ and $0 \leq u(x, t, \varphi) \leq$ 1 for all $x \in R$ and $t \in[0, \infty)$, we then have

$U(-H+x)-\delta \leq u(x, t, \varphi) \leq U(H+x)+\delta, \quad$ for all $x \in R \quad$ and $t \in[T-\tau, T]$.

Let $\xi_{0}=-H+c T, h_{0}=2 H>0$. Then (3.32) implies that

$U\left(x-c T+\xi_{0}\right)-\delta \leq u_{T}(x, \varphi)(s) \leq U\left(x-c T+\xi_{0}+h_{0}\right)+\delta, \quad s \in[-\tau, 0], \quad x \in R$.

Let $\xi=\xi_{0}+\sigma_{0} \delta\left(1-e^{\beta_{0} \tau}\right)-|c| \tau$ and $h=h_{0}-2 \sigma_{0} \delta\left(1-e^{\beta_{0} \tau}\right)+2|c| \tau>0$. Then by (3.33), we have that for any $s \in[-\tau, 0]$ and $x \in R$,

$$
\begin{aligned}
& w_{0}^{-}(x,-c T+\xi, \delta)(s) \\
& \quad=U\left(x-c s-c T+\xi-\sigma_{0} \delta\left(1-e^{-\beta_{0} s}\right)\right)-\delta e^{-\beta_{0} s} \\
& \quad \leq U\left(x+|c| \tau-c T+\xi-\sigma_{0} \delta\left(1-e^{\beta_{0} \tau}\right)\right)-\delta \\
& \quad=U\left(x-c T+\xi_{0}\right)-\delta \leq u_{T}(x, \varphi)(x),
\end{aligned}
$$

and

$$
\begin{aligned}
& w_{0}^{+}(x,-c T+\xi+h, \delta)(s) \\
& \quad=U\left(x-c s-c T+\xi+h+\sigma_{0} \delta\left(1-e^{-\beta_{0} s}\right)\right)+\delta e^{-\beta_{0} s} \\
& \quad \geq U\left(x-|c| \tau-c T+\xi+h+\sigma_{0} \delta\left(1-e^{\beta_{0} \tau}\right)\right)+\delta \\
& \quad=U\left(x-c T+\xi_{0}+h_{0}\right)+\delta \geq u_{T}(x, \varphi)(s) .
\end{aligned}
$$

It then follows that

$$
w_{0}^{-}(x,-c T+\xi, \delta)(s) \leq u_{T}(x, \varphi)(s) \leq w_{0}^{+}(x,-c T+\xi+h, \delta)(s) .
$$

This completes the proof.

Now we are in a position to prove the main results in this section. 
Theorem 3.1 Assume that (2.1) has a monotone traveling wave solution $U(x-c t)$. Then $U(x-c t)$ is globally asymptotically stable with phase shift in the sense that there exists $k>0$ such that for any $\varphi \in[0,1]_{C}$ with

$$
\liminf _{x \rightarrow \infty} \min _{s \in[-\tau, 0]} \varphi(x, s)>a^{+}, \quad \limsup _{x \rightarrow-\infty} \max _{s \in[-\tau, 0]} \varphi(x, s)<a^{-},
$$

the solution $u(x, t, \varphi)$ of (2.1) satisfies

$$
|u(x, t, \varphi)-U(x-c t+\xi)| \leq K e^{-k t}, \quad x \in R, t \geq 0,
$$

for some $K=K(\varphi)>0$ and $\xi=\xi(\varphi) \in R$.

Proof. Let $\beta_{0}, \sigma_{0}, \bar{\delta}$ be as in Lemma 2.1 with $\beta_{0}$ chosen such that $3 e^{\beta_{0} \tau}<4$, and then let $\epsilon^{*}$ be as in Lemma 3.1 with $\epsilon^{*}$ chosen such that $\left(4-3 e^{\beta_{0} \tau}\right) \sigma_{0} \cdot \epsilon^{*}<$ 1. We further choose a $0<\delta^{*}<\min \left(\frac{\delta_{0}}{2}, \bar{\delta}, \frac{1}{\sigma_{0}}\right)$ such that

$$
1>k^{*}:=\left(4-3 e^{\beta_{0} \tau}\right) \sigma_{0} \epsilon^{*}-3 e^{\beta_{0} \tau} \sigma_{0} \delta^{*}>0,
$$

and then fix a $t^{*} \geq \tau+1$ such that

$$
e^{-\beta_{0}\left(t^{*}-\tau-1\right)}\left(1+\epsilon^{*} / \delta^{*}\right)<1-k^{*} .
$$

We first prove the following two claims.

Claim 1 There exist $T^{*}=T^{*}(\varphi)>0, \xi^{*}=\xi^{*}(\varphi) \in R$ such that

$$
\begin{gathered}
w_{0}^{-}\left(x,-c T^{*}+\xi^{*}, \delta^{*}\right)(s) \leq u_{T^{*}}(x, \varphi)(s) \leq w_{0}^{+}\left(x,-c T^{*}+\xi^{*}+1, \delta^{*}\right)(s), \\
s \in[-\tau, 0], x \in R .
\end{gathered}
$$

Indeed, by Lemma 3.2, there exist $T=T(\varphi)>0, \xi=\xi(\varphi) \in R$ and $h=h(\varphi)>0$ such that

$$
\begin{gathered}
w_{0}^{-}\left(x,-c T+\xi, \delta^{*}\right)(s) \leq u_{T}(x, \varphi)(s) \leq w_{0}^{+}\left(x,-c T+\xi+h, \delta^{*}\right)(s), \\
s \in[-\tau, 0], \quad x \in R .
\end{gathered}
$$

If $h \leq 1$, (3.34) follows immediately from the monotonicity of $U(\cdot)$. Then we assume that $h>1$ and let

$$
N=\max \left\{m ; m \text { is a nonnegative integer and } m k^{*}<h\right\} .
$$


Since $0<k^{*}<1$ and $h>1$, we have $N \geq 1, N k^{*}<h \leq(N+1) k^{*}$ and hence $0<h-N k^{*} \leq(N+1) k^{*}-N k^{*}=k^{*}<1$. Clearly, $\bar{h}:=\min (1, h)=1$. By (3.35), Lemma 3.1 and the choice of $t^{*}$ and $k^{*}$, it then follows that

$$
\begin{aligned}
w_{0}^{-}\left(x,-c\left(T+t^{*}\right)+\hat{\xi}\left(T+t^{*}\right), \hat{\delta}\left(T+t^{*}\right)(s) \leq\right. & u_{T+t^{*}}(x, \varphi)(s) \\
\leq & w_{0}^{+}\left(x,-c\left(T+t^{*}\right)+\hat{\xi}\left(T+t^{*}\right)\right. \\
& +\hat{h}\left(T+t^{*}\right), \hat{\delta}\left(T+t^{*}\right)(s), \\
& s \in[-\tau, 0], x \in R, \quad(3.36)
\end{aligned}
$$

where

$$
\begin{aligned}
& \hat{\xi}\left(T+t^{*}\right) \in\left[\xi-\sigma_{0} \delta^{*}-2 \sigma_{0}\left(\delta^{*}+\epsilon^{*}\right) e^{\beta_{0} \tau}, \xi+h+\sigma_{0} \delta^{*}\right], \\
& \hat{\delta}\left(T+t^{*}\right)=\left(\delta^{*} e^{-\beta_{0}}+\epsilon^{*}\right) e^{-\beta_{0}\left(t^{*}-\tau-1\right)} \leq\left(1-k^{*}\right) \delta^{*}<\delta^{*}, \\
& 0 \leq \hat{h}\left(T+t^{*}\right) \leq h+\left(3 e^{\beta_{0} \tau}-4\right) \sigma_{0} \epsilon^{*}+3 e^{\beta_{0} \tau} \sigma_{0} \delta^{*}=h-k^{*} .
\end{aligned}
$$

Repeating the same process $N$ times, we have that (3.36), with $T+t^{*}$ replaced by $T+N t^{*}$, holds for some $\hat{\xi} \in R, 0<\hat{\delta} \leq \delta^{*}$ and $0 \leq \hat{h} \leq h-N k^{*}<1$. Let $T^{*}=T+N t^{*}, \xi^{*}=\hat{\xi}$. Again, by the monotonicity of $\bar{U}(\cdot),(3.34)$ then follows.

Claim 2 Let $p=2 \sigma_{0} \delta^{*}+2 \sigma_{0}\left(\delta^{*}+\epsilon^{*}\right) e^{\beta_{0} \tau}+1, T_{m}=T^{*}+m t^{*}, \delta_{m}^{*}=\left(1-k^{*}\right)^{m} \delta^{*}$ and $h_{m}=\left(1-k^{*}\right)^{m}, m \geq 0$. Then there exists a sequence $\left\{\xi_{m}\right\}_{m=0}^{\infty} \subset R$ with $\xi_{0}=\xi^{*}$ such that

$$
\left|\xi_{m+1}-\xi_{m}\right| \leq p h_{m}, \quad m \geq 0
$$

and

$$
\begin{aligned}
w_{0}^{-}\left(x,-c T_{m}+\xi_{m}, \delta_{m}^{*}\right)(s) \leq & u_{T_{m}}(x, \varphi)(s) \\
\leq & w_{0}^{+}\left(x,-c T_{m}+\xi_{m}+h_{m}, \delta_{m}^{*}\right)(s), \\
& s \in[-\tau, 0], \quad x \in R, m \geq 0 .
\end{aligned}
$$

In fact, Claim 1 implies that (3.38) holds for $m=0$. Now suppose that (3.38) holds for some $m=l \geq 0$. By Lemma 3.1 with $T=T_{l}, \xi=\xi_{l}, h=h_{l}$, $\delta=\delta_{l}^{*}$ and $t=T_{l}+t^{*}=T_{l+1}\left(\right.$ since $\left.t^{*} \geq \tau+1\right)$, we then have

$$
\begin{gathered}
w_{0}^{-}\left(x,-c T_{l+1}+\hat{\xi}, \hat{\delta}\right)(s) \leq u_{T_{l+1}}(x, \varphi)(s) \leq w_{0}^{+}\left(x,-c T_{l+1}+\hat{\xi}+\hat{h}, \hat{\delta}\right)(s), \\
s \in[-\tau, 0], \quad x \in R
\end{gathered}
$$


where

$$
\begin{aligned}
\hat{\xi} & \in\left[\xi_{l}-\sigma_{0} \delta_{l}^{*}-2 \sigma_{0}\left(\delta_{l}^{*}+\epsilon^{*} h_{l}\right) e^{\beta_{0} \tau}, \xi_{l}+h_{l}+\sigma_{0} \delta_{l}^{*}\right] \\
\hat{\delta} & =\left(\delta_{l} e^{-\beta_{0}}+\epsilon^{*} h_{l}\right) e^{-\beta_{0}\left(T_{l+1}-T_{l}-\tau-1\right)} \\
& \leq\left(1-k^{*}\right)^{l} \cdot \delta^{*}\left[\left(1+\frac{\epsilon^{*}}{\delta^{*}}\right) e^{-\beta_{0}\left(t^{*}-\tau-1\right)}\right] \\
& \leq\left(1-k^{*}\right)^{l} \cdot \delta^{*}\left(1-k^{*}\right)=\delta_{l+1}^{*}, \\
\hat{h} & \leq h_{l}-\left(4-3 e^{\beta_{0} \tau}\right) \sigma_{0} \epsilon^{*} h_{l}+3 e^{\beta_{0} \cdot \tau} \sigma_{0} \delta_{l} \\
& =\left(1-k^{*}\right)^{l} \cdot\left[1-\left(4-3 e^{\beta_{0} \tau}\right) \sigma_{0} \epsilon^{*}+3 e^{\beta_{0} \tau} \sigma_{0} \delta^{*}\right] \\
& =\left(1-k^{*}\right)^{l+1}=h_{l+1} .
\end{aligned}
$$

We choose $\xi_{l+1}=\hat{\xi}$. Then

$$
\left|\xi_{l+1}-\xi_{l}\right| \leq\left|\xi_{l}+h_{l}+\sigma_{0} \delta_{l}^{*}-\left(\xi_{l}-\sigma_{0} \delta_{l}^{*}-2 \sigma_{0}\left(\delta_{l}^{*}+\epsilon^{*} h_{l}\right) \cdot e^{\beta_{0} \tau}\right)\right|=p \cdot h_{l} .
$$

It then follows that (3.37) holds for $m=l$, and (3.38) holds for $m=l+1$. By induction, (3.37) and (3.38) hold for all $m \geq 0$.

For each $m \geq 0$, by (3.38) and Theorem 2.1, it follows that for all $t \geq T_{m}$, $x \in R$,

$$
\begin{aligned}
& U\left(x-c t+\xi_{m}-\sigma_{0} \delta_{m}^{*}\left(1-e^{-\beta_{0}\left(t-T_{m}\right)}\right)\right)-\delta_{m}^{*} e^{-\beta_{0}\left(t-T_{m}\right)} \leq u(x, t, \varphi) \\
& \quad \leq U\left(x-c t+\xi_{m}+h_{m}+\sigma_{0} \delta_{m}^{*}\left(1-e^{-\beta_{0}\left(t-T_{m}\right)}\right)\right)+\delta_{m}^{*} e^{-\beta_{0}\left(t-T_{m}\right)}
\end{aligned}
$$

For any $t \geq T^{*}$, let $m=\left[\frac{t-T^{*}}{t^{*}}\right] \geq 0$ be the largest integer not greater than $\frac{t-T^{*}}{t^{*}}$, and define $\delta(t)=\delta_{m}^{*}, \xi(t)=\xi_{m}-\sigma_{0} \delta_{m}^{*}$ and $h(t)=h_{m}+2 \sigma_{0} \delta_{m}^{*}$, then we have $T_{m}=T^{*}+m t^{*} \leq t<T^{*}+(m+1) t^{*}=T_{m+1}$. By (3.40), it follows that for all $t \geq T^{*}, x \in R$,

$$
U(x-c t+\xi(t))-\delta(t) \leq u(x, t, \varphi) \leq U(x-c t+\xi(t)+h(t))+\delta(t)
$$

Moreover, we have

$$
\begin{aligned}
\delta(t)=\delta_{m}^{*} & =\left[1-k^{*}\right]^{m} \delta^{*} \leq \delta^{*} \exp \left\{\left(\frac{t-T^{*}}{t^{*}}-1\right) \ln \left(1-k^{*}\right)\right\}, \quad t \geq T^{*}, \\
h(t) & =h_{m}+2 \sigma_{0} \delta_{m}^{*}=\left(1+2 \sigma_{0} \delta^{*}\right)\left(1-k^{*}\right)^{m} \\
& \leq\left(1+2 \sigma_{0} \delta^{*}\right) \exp \left\{\left(\frac{t-T^{*}}{t^{*}}-1\right) \ln \left(1-k^{*}\right)\right\}, \quad t \geq T^{*},(3.43)
\end{aligned}
$$


and for any $r \geq t \geq T^{*}$, by (3.37),

$$
\begin{aligned}
|\xi(r)-\xi(t)| & =\left|\xi_{m}-\sigma_{0} \delta_{m}^{*}-\left(\xi_{n}-\sigma_{0} \delta_{n}^{*}\right)\right| \\
& \leq\left|\xi_{m}-\xi_{n}\right|+\sigma_{0} \delta_{m}^{*}+\sigma_{0} \delta_{n}^{*} \\
& \leq \sum_{l=n}^{m-1}\left|\xi_{l+1}-\xi_{l}\right|+2 \sigma_{0} \delta_{n}^{*} \\
& \leq \sum_{l=n}^{m-1} p \cdot h_{l}+2 \sigma_{0} \delta_{n}^{*} \\
& \leq \frac{p h_{n}}{1-\left(1-k^{*}\right)}+2 \sigma_{0} \delta_{n}^{*} \\
& =q \cdot \delta(t),
\end{aligned}
$$

where $m=\left[\frac{r-T^{*}}{t^{*}}\right] \geq n=\left[\frac{t-T^{*}}{t^{*}}\right]$, and $q=\frac{p}{k^{*} \delta^{*}}+2 \sigma_{0}$. Clearly, (3.44) implies that $\xi(\infty)=\lim _{t \rightarrow \infty} \xi(t)$ exists and

$$
|\xi(\infty)-\xi(t)| \leq q \delta(t), \quad t \geq T^{*} .
$$

Then

$$
|\xi(t)-\xi(\infty)| \leq q \cdot \delta^{*} \exp \left\{\left(\frac{t-T^{*}}{t^{*}}-1\right) \ln \left(1-k^{*}\right)\right\}, \quad t \geq T^{*} .
$$

Therefore, by defining $k=-\frac{1}{t^{*}} \ln \left(1-k^{*}\right)>0$ and combining (3.41), (3.42), (3.43) and (3.45), we obtain the assertion of the theorem.

This completes the proof.

Theorem 3.2 Every monotone traveling wave solution of (2.1) is Liapunov stable. If (2.1) has a monotone traveling wave solution $U(x-c t)$, then the traveling wave solutions of (2.1) are unique up to a translation in the sense that for any traveling wave solution $\bar{U}(x-\bar{c} t)$ with $0 \leq \bar{U}(\xi) \leq 1, \xi \in R$, we have $\bar{c}=c$ and $\bar{U}(\cdot)=U\left(\xi_{0}+\cdot\right)$ for some $\xi_{0}=\xi_{0}(\bar{U}) \in R$.

Proof. Let $U(x-c t)$ be a monotone traveling wave solution of (2.1). By the uniform continuity of $U(\cdot)$ on $R$, it follows that for any $\epsilon>0$, there exists a $\delta_{1}=\delta_{1}(\epsilon)>0$ such that for all $|y| \leq \delta_{1}$,

$$
|U(x-c t+y)-U(x-c t)|<\frac{\epsilon}{2}, \quad x \in R, t \geq 0 .
$$


We then further choose a $\delta=\delta(\epsilon)>0$ such that $\delta<\min \left(\frac{\epsilon}{2}, \frac{\delta_{1} \cdot e^{-\beta_{0} \tau}}{\sigma_{0}}, \bar{\delta}\right)$, where $\beta_{0}, \sigma_{0}$ and $\bar{\delta}$ are as in Lemma 2.1. For any $\varphi \in C([-\tau, 0], X)$ with $|\varphi(x, s)-U(x-c s)|<\delta$ for $s \in[-\tau, 0]$ and $x \in R$, we have

$$
\begin{aligned}
& U\left(x-c s+\sigma_{0} \delta\left(1-e^{\beta_{0} \tau}\right)-\sigma_{0} \delta\left(1-e^{-\beta_{0} s}\right)\right)-\delta e^{-\beta_{0} s} \\
& \leq \varphi(x, s) \leq U\left(x-c s+\sigma_{0} \delta\left(e^{\beta_{0} \tau}-1\right)\right. \\
& \left.\quad+\sigma_{0} \delta\left(1-e^{-\beta_{0} s}\right)\right)+\delta e^{-\beta_{0} s}, \quad s \in[-\tau, 0], x \in R .
\end{aligned}
$$

By Lemma 2.1 and Theorem 2.1, it then follows that

$$
\begin{aligned}
U\left(x-c t+\sigma_{0} \delta\left(1-e^{\beta_{0} \tau}\right)-\sigma_{0} \delta\left(1-e^{-\beta_{0} t}\right)\right)-\delta e^{-\beta_{0} t} \leq u(x, t, \varphi) \\
\leq \\
\quad U\left(x-c t+\sigma_{0} \delta\left(e^{\beta_{0} \tau}-1\right)+\sigma_{0} \delta\left(1-e^{-\beta_{0} t}\right)\right) \\
\quad+\delta e^{-\beta_{0} t}, x \in R, t \geq 0 .
\end{aligned}
$$

By the choice of $\delta=\delta(\epsilon)$, we have that for all $t \geq 0$,

$$
\begin{aligned}
\left|\sigma_{0} \delta\left(1-e^{\beta_{0} \tau}\right)-\sigma_{0} \delta\left(1-e^{-\beta_{0} t}\right)\right| & \leq \sigma_{0} \delta\left(e^{\beta_{0} \tau}-1\right)+\sigma_{0} \delta\left(1-e^{-\beta_{0} t}\right) \\
& \leq \sigma_{0} \delta e^{\beta_{0} \tau}<\delta_{1}(\epsilon)
\end{aligned}
$$

and

$$
\left|\sigma_{0} \delta\left(e^{\beta_{0} \tau}-1\right)+\sigma_{0} \delta\left(1-e^{-\beta_{0} t}\right)\right| \leq \sigma_{0} \delta e^{\beta_{0} \tau}<\delta_{1}(\epsilon) .
$$

Then, by (3.46) and (3.48), it follows that $U(x-c t)-\epsilon \leq u(x, t, \varphi) \leq$ $U(x-c t)+\epsilon, x \in R, t \geq 0$. That is, $|u(x, t, \varphi)-U(x-c t)|<\epsilon, x \in R$, $t \geq 0$. Therefore $U(x-c t)$ is Liapunov stable.

To prove the uniqueness, we let $U(x-c t)$ be the given monotone traveling wave solution, and let $\bar{U}(x-\bar{c} t)$ be any traveling wave solution of (2.1) with $0 \leq \bar{U} \leq 1$ on $R$. Since $\lim _{x \rightarrow \infty} \bar{U}(x-\bar{c} s)=1$ and $\lim _{x \rightarrow-\infty} \bar{U}(x-\bar{c} s)=0$ uniformly for $s \in[-\tau, 0]$, we have

$$
\begin{aligned}
& \liminf _{x \rightarrow \infty} \min _{s \in[-\tau, 0]} \bar{U}(x-\bar{c} s)>a^{+} \quad \text { and } \\
& \limsup _{x \rightarrow-\infty} \max _{s \in[-\tau, 0]} \bar{U}(x-\bar{c} s)<a^{-} .
\end{aligned}
$$

Then, by Theorem 3.1, there exists $K_{0}=K_{0}(\bar{U})>0$ and $\xi_{0}=\xi_{0}(\bar{U}) \in R$ such that

$$
\left|\bar{U}(x-\bar{c} t)-U\left(x-c t+\xi_{0}\right)\right| \leq K_{0} e^{-k t}, \quad x \in R, \quad t \geq 0 .
$$


Let $\bar{\xi} \in R$ be such that $0<\bar{U}(\bar{\xi})<1$, and define $L(\bar{\xi}):=\{(x, t) ; x \in R, t \geq$ $0, x-\bar{c} t=\bar{\xi}\}$. By $(3.50)$, it then follows that

$$
\begin{gathered}
U\left(\bar{\xi}+\xi_{0}+(\bar{c}-c) t\right)-K_{0} e^{-k t} \leq \bar{U}(\bar{\xi}) \leq U\left(\bar{\xi}+\xi_{0}+(\bar{c}-c) t\right)+K_{0} e^{-k t} \\
\quad \text { for all }(x, t) \in L(\bar{\xi})
\end{gathered}
$$

Since $U(\infty)=1$ and $U(-\infty)=0$, letting $t \rightarrow \infty$ in (3.51), we obtain that $\bar{c} \leq c$ from the first inequality and that $\bar{c} \geq c$ from the second inequality. Then $\bar{c}=c$. For any $\xi \in R$, again by (3.50), we then have

$$
\left|\bar{U}(\xi)-U\left(\xi+\xi_{0}\right)\right| \leq K_{0} e^{-k t}, \quad \text { for all }(x, t) \in L(\xi) .
$$

Therefore, letting $t \rightarrow \infty$ in (3.52), we get $\bar{U}(\xi)=U\left(\xi+\xi_{0}\right)$, for all $\xi \in R$, that is, $\bar{U}(\cdot)=U\left(\xi_{0}+\cdot\right)$.

This completes the proof.

Remark 3.1 For the typical Huxley nonlinearity:

$$
f(u, v)= \begin{cases}u(1-u)(v-a) & \text { for } 0 \leq u \leq 1, v \in R \\ u(1-u)(u-a) & \text { otherwise }\end{cases}
$$

with $0<a<1$, let $\hat{f}: I^{2} \rightarrow R$ be a smooth extension of $f:[0,1]^{2} \rightarrow R$ such that (H1) and (H2) hold for $\hat{f}$. Clearly, [7, Corollary 5] implies that $[0,1]_{C}$ is positively invariant for (2.1) with $f$ replaced by $\hat{f}$. It then follows that Theorems 3.1 and 3.2 hold for (2.1) with the Huxley nonlinearity.

Remark 3.2 With some additional assumptions on $f(\cdot, \cdot)$, Schaaf [13] proved the existence of monotone traveling wave solution of (2.1) and uniqueness of the wave speeds. By Theorems 3.1 and 3.2 we further conclude that this monotone traveling wave solution is globally and exponentially asymptotically stable with phase shift, that all traveling waves are unique up to translation and that every traveling wave solution is Liapunov stable.

\section{References}

[1] X. Chen, Existence, uniqueness, and asymptotic stability of traveling waves in nonlocal evolution equations, Advances in Differential Equations, 2 (1997), 125-160. 
[2] D. Daners and P. K. Medina, Abstract Evolution Equations, Periodic Problems and Applications, Pitman Research Notes in Mathematics, Ser. 279, Longman Sci. \& Tech., 1992.

[3] P. C. Fife and J. B. McLeod, The approach of solutions of nonlinear diffusion equations to traveling wave solutions, Arch. Rational Mech. Anal., 65 (1977), 335-361.

[4] H. I. Freedman and X.-Q. Zhao, Global asymptotics in some quasimonotone reaction-diffusion systems with delays, J. Diff. Eqs., 137 (1997), 340-362.

[5] K. Gopalsamy, Stability and Oscillations in Delay Differential Equations of Population Dynamics, Kluwer Academic Publishers, 1992.

[6] M. Hirsch, H. Hanish and J.-P. Gabriel, Differential equation model of some parasitic infections: methods for the study of asymptotic behavior, Comm. Pure. Appl. Math., 38(1985), 733-753.

[7] R. H. Martin and H. L. Smith, Abstract functional differential equations and reaction-diffusion systems, Trans. Amer. Math. Soc., 321 (1990), $1-44$.

[8] R. H. Martin and H. L. Smith, Reaction-diffusion systems with time delays: Monotonicity, invariance, comparison and convergence, J. Reine Angew. Math., 413 (1991), 1-35.

[9] T. Ogiwara and H. Matano, Monotonicity and convergence result in order-preserving systems in the presence of symmetry, Discrete and Continuous Dynamical Systems, 5(1999), 1-34.

[10] A. Pazy, Semigroups of Linear Operators and Applications to Partial Differential Equations, Springer-Verlag, New York, 1983.

[11] J.-M. Roquejoffre, D. Terman and V. A. Volpert, Global stability of traveling fronts and convergence towards stacked families of waves in monotone parabolic systems, SIAM J. Math. Anal., 27 (1996), 12611269 .

[12] W. Shen, Traveling waves in time almost periodic structures governed by bistable nonlinearities I and II, J. Diff. Eqs., to appear. 
[13] K. W. Schaaf, Asymptotic behavior and traveling wave solutions for parabolic functional differential equations, Trans. Amer. Math. Soc., 302 (1987), 587-615.

[14] H. L. Smith, Monotone dynamical systems, An introduction to the theory of competitive and cooperative systems, Math. Surveys and Monographs, Vol. 41, Amer. Math. Soc., Providence, 1995.

[15] C. C. Travis and G. F. Webb, Existence and stability for partial functional differential equations, Trans. Amer. Math. Soc., 200 (1974), 395418.

[16] A. I. Volpert, Vitaly A. Volpert and Vladimir A. Volpert, Traveling Wave Solutions of Parabolic Systems, Translation of Math. Monographs, Amer. Math. Soc., Providence, 1994.

[17] J. Wu, Theory and Applications of Partial Functional Differential Equations, Applied Math. Sci., Vol. 119, Springer-Verlag, New York, 1996.

[18] X. Zou and J. Wu, Existence of traveling wave fronts in delayed reactiondiffusion systems via the monotone iteration method, Proc. Amer. Math. Soc., 125 (1997), 2589-2598. 